\title{
O papel do aluno de graduação em Medicina no atendimento a pacientes de enfermarias de longa permanência de um hospital-escola
}

PALAVRAS-CHAVE:

Relações Médico-Paciente;

Estudantes de Medicina;

Relações Hospital-Paciente;

Satisfação do Paciente.

\section{KEY-WORDS:}

Physician-Patient Relations;

Students, Medical;

Hospital-Patient Relations;

Patient Satisfaction.

Recebido em: 01/11/2006

Reencaminhado em: 15/03/2007

Reencaminhado em: 01/08/2007

Reencaminhado em: 26/08/2007

Aprovado em: 11/10/2007

\section{The role of the graduation Medical student in the care of patients in long permanence ward of a medical school hospital}

Paulo César Brandão Veiga Jardim ${ }^{1}$

Nayara Gomes Silveira da Costa ${ }^{1}$

Priscilla Caixeta de Oliveira Verena Nunes e Silva ${ }^{1}$

Yana de Sousa Rabelo ${ }^{1}$

\section{RESUMO}

Verificar a percepção que os pacientes internados em um Hospital Escola (HC-UFG) têm da presença dos alunos de medicina no atendimento prestado a eles e observar outros fatores relacionados à satisfação com o atendimento. MÉTODO: Critério de inclusão: internação há menos de um ano em outro Hospital não Escola. Excluídos: Menores de 18 anos e incapazes de colaborar. Aplicado questionário, por alunas da Faculdade de Medicina da UFG, com informações sobre a internação, privacidade, comparação com outros hospitais e avaliação da presença dos alunos. Utilizados estatística descritiva, teste de Wilcoxon, c e Regra de Sinais de Descartes. RESULTADOS: Avaliados 96 pacientes. A média das notas do HC-UFG foi 9,01 $\pm 1,5$, a dos

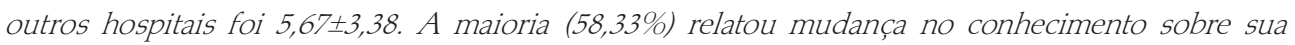
doença após a internação no HC-UFG. A presença dos alunos foi avaliada como boa ou muito boa por 91,58\% e despertou sentimentos positivos em 84,54\% dos entrevistados. As aulas ao redor dos leitos foram consideradas agradáveis por 69,79\% deles. CONCLUSÃO: O aluno tem papel relevante no atendimento prestado no Hospital Escola. Contribui para maior confiança no serviço, desperta maior segurança e alegria nos pacientes, torna o ambiente mais acolhedor e atua como disseminador de conhecimentos.

\begin{abstract}
To investigate the patients' perception of the students in their care in a long permanence ward of the School Hospital of the Federal University of Goiás (HC-UFG) and to observe another factors related to these patients' satisfaction. METHOD: Inclusion criterion: length of hospital stay of no longer than one and a half year at a non-school hospital. Individuals under 18 years of age and unable to collaborate were excluded. Participants responded to a questionnaire, applied by medical students of UFG, with information regarding hospitalization, privacy, comparison with other hospitals and evaluation of students' presence. Descriptive statistical analysis, Wilcoxon's Test, $c^{2}$ and Descartes' Rule of Sign were used. RESULTS: 96 patients were evaluated. The average

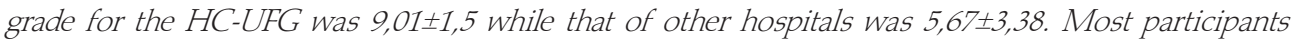
$(58,33 \%)$ reported a change regarding knowledge of their disease after hospitalization at the HC-
\end{abstract}


UFG. The presence of students was deemed good or very good by $91,58 \%$ and yielded positive feelings in $84,54 \%$ of the interviewees. Bedside-held classes were considered pleasant by 69,79\% of the participants. CONCLUSION: The student has a relevant role in caregiving at the School Hospital. It further contributes to more reliability on the service, gives rise to the more security and happiness among patients, makes the environment cozier and acts as knowledge disseminator.

\section{INTRODUÇÃO}

A cada ano, novos alunos de Medicina iniciam seus primeiros contatos com pacientes nos serviços de atendimento primário à saúde e em hospitais universitários (HU). Nestes, há uma intensificação dos contatos com o início da parte clínica do curso, na disciplina de Semiologia Médica, que, na Universidade Federal de Goiás (UFG), ocorre no quarto semestre.

Os estudantes se vêem diante de uma situação nunca vivida. Estabelecer uma relação com o primeiro paciente sem acompanhamento direto é, com certeza, um evento gerador de conflitos.

Além da insegurança habitual, comum a todos os aprendizes, o estudante de Medicina encara a dificuldade de lidar com uma pessoa enferma, que, por sua condição, encontra-se abalada física e emocionalmente.

Incertezas permeiam esse primeiro contato: como chegar até meu paciente? Que benefício proporciono a ele? Será que o paciente está se sentindo incomodado? Estou interrompendo o seu tempo de descanso? E se ele não quiser conversar comigo?

Estes e muitos outros questionamentos aumentam ainda mais a insegurança dos iniciantes da prática médica. Como bem ilustra Martins ${ }^{1}$ (p. 185):

O iniciante no contato com o paciente apresenta uma série de temores, perfeitamente justificáveis, que vão desde a sua insegurança pessoal, em relação a conhecimentos que devem ser postos em prática, até um certo tipo de reação paranóide, onde nos passa pela mente: o que aquela pessoa a nossa frente estará pensando ou imaginando de nós?

Uma preocupação extra para quem inicia suas atividades no hospital-escola é saber o que o paciente sente nesse momento do relacionamento e qual a impressão dele ao término da entrevista. Conhecer mais de perto este amplo espectro de percepções vivenciadas pelo paciente é de grande relevância, pois propicia ao estudante colocar acima de tudo a condição humana de seu ato.

A ocasião dos primeiros encontros é favorável ao desenvolvimento de uma boa relação médico-paciente, que acompanhará o futuro médico por toda a vida profissional. É nesse momento que o aluno gasta maior tempo e atenção em cada encontro, já que, no decorrer do curso, ele passa a dedicar menos tempo a um só paciente, preocupando-se mais com a aplicação dos conhecimentos técnicos que está adquirindo. Segundo Porto ${ }^{2}$ (p. 16):

[...] mais importante que diagnosticar, receitar ou operar é cuidar do paciente. E isso você (o estudante) pode fazer até melhor do que o Residente ou o Professor que é especialista na doença do seu paciente.

O reconhecimento pelo aluno de que sua presença agrada o paciente e de que ele é capaz de melhorar a qualidade da internação poderia contribuir de maneira significativa para minimizar as angústias despertadas nos iniciantes.

Como participa das atividades do hospital-escola, o estudante provavelmente terá influência na satisfação dos pacientes com o serviço. No entanto, outras variáveis estão envolvidas nesse processo. Algumas são subjetivas: a expectativa do paciente em relação ao tratamento, a aceitação da doença, a rede social e aspectos socioculturais vivenciados por ele. Outras são objetivas e incluem a limpeza do ambiente, alimentação, estrutura física, assistência prestada pelo médico responsável, pelas enfermeiras e por toda a equipe hospitalar, assim como recursos diagnósticos e de tratamento.

A perda de privacidade dos pacientes internados nas enfermarias do hospital universitário, por onde inúmeras pessoas circulam diariamente para colher informações e fazer exames, também pode influenciar a qualidade de sua estada.

Nota-se que, com o passar do tempo da internação, alguns pacientes adquirem maior gama de conhecimentos sobre sua doença. Este fato talvez seja mais um dos fatores que contribui para a avaliação do atendimento.

Poucos trabalhos foram desenvolvidos sobre a interação aluno-paciente e a possível influência desta relação na satisfação dos pacientes com o serviço. A maioria destes, como o desenvolvido por uma equipe da Johns Hopkins University School ${ }^{3}$ e outro pela Harvard Medical School and Harvard Pilgrim Health Care ${ }^{4}$, avalia a interferência da presença de alunos, porém em atendimentos ambulatoriais. Os pacientes internados, que convivem com estudantes desde o início do aprendizado médico, não foram considerados nestas investigações. Por isto, julgamos ser de grande relevância pesquisar o grau de satisfação dos pacientes acamados relacionado à convivência diária com estudantes de Medicina.

O objetivo principal desta investigação foi verificar o papel dos alunos de Medicina no atendimento prestado aos pacientes internados em um hospital universitário e observar os fatores relacionados à satisfação do paciente com o atendimento. Espera-se, ainda, que, além de quantificar esses fatores, o estudo atue como orientador e facilitador dos primeiros contatos dos estudantes de Medicina com seus pacientes. 


\section{CASUÍSTICA E MÉTODO}

\section{Local e grupo pesquisado}

O estudo foi sediado no Hospital das Clínicas da Universidade Federal de Goiás (HC-UFG), onde os alunos da Faculdade de Medicina recebem parte de sua educação médica. O HCUFG é um hospital de abrangência regional que atende pacientes provenientes de Goiânia e do interior dos estados de Goiás, Tocantins, Bahia e outros, em especial da Região Norte. O hospital admite, anualmente, grande número de pacientes, sendo que em 2004 foram internadas 10.449 pessoas.

Os critérios de inclusão foram: pacientes maiores de 18 anos, internados nas enfermarias do HC-UFG, que tivessem tido outra internação há menos de um ano em outro hospital (não escola) e que concordassem em participar da pesquisa assinando um termo de consentimento livre e esclarecido. A partir do número mensal de internações, foi definida uma amostra de 90 pacientes como representativa dos indivíduos internados. Foram entrevistados pacientes em nove enfermarias da Clínica Médica, em dez enfermarias da Clínica Cirúrgica e em três da Unidade de Emergência. Excluíram-se aqueles que por qualquer motivo fossem incapazes de colaborar.

\section{Instrumento utilizado}

Os dados foram colhidos por meio de um questionário elaborado e aplicado por alunas do terceiro ano do curso de Medicina. As entrevistas foram realizadas de 14 de abril a 14 de maio de 2005, após aprovação do Comitê de Ética em Pesquisa Médica Humana e Animal do Hospital das Clínicas da Universidade Federal de Goiás.

O questionário é composto por dados demográficos, informações a respeito da internação e privacidade do paciente, aspectos comparativos entre o HC-UFG e os outros hospitais não universitários, e avaliação dos entrevistados quanto à presença dos alunos no ambiente hospitalar.

\section{Critério de classificação das variáveis numéricas}

As variáveis que exigiram atribuição de notas pelos entrevistados foram reclassificadas segundo este critério: as notas entre 0 e 4 foram consideradas ruins; 5 ou 6 , regulares; 7 ou 8, boas; e 9 ou 10, muito boas.

\section{Análise estatística}

O banco de dados, com todas as variáveis incluídas no estudo, foi criado com o uso do programa Excel-Microsoft Office 2000.

Foi utilizado o teste de Wilcoxon para a comparação das notas médias obtidas pelos hospitais avaliados. A Regra de
Sinais de Descartes foi utilizada na análise das variáveis que mediram a qualidade do serviço oferecido pelo HC-UFG e da estada nesse hospital, e ainda a mudança no conhecimento da doença. Para verificar a ocorrência de associação entre as variáveis estudadas, usamos o teste do qui-quadrado.

\section{RESULTADOS}

\section{Caracterização da amostra}

Foram entrevistados 96 pacientes com idade média de 45,5 $\pm 15,0$ anos. O sexo feminino correspondeu a $53,13 \%$ dos entrevistados, e o masculino a $45,83 \%$. Os casados representaram $65,63 \%$ da amostra, os solteiros $21,88 \%$, e os demais somaram $12,5 \%$. A maioria dos pacientes morava no estado de Goiás, e não houve diferença entre os provenientes de Goiânia $(44,79 \%)$ e do interior do estado $(44,79 \%)$. As últimas internações dos pacientes ocorreram em 21,88\% dos casos fora da cidade de Goiânia; entre os internados na capital, 21,88\% foram internados na rede pública e $55,21 \%$ nos hospitais conveniados com o SUS. A maior parte dos entrevistados foi internada menos de três vezes no último ano, sendo que $12,5 \%$ dos pacientes já estiveram internados mais de três vezes no Hospital das Clínicas da UFG.

Em relação ao tempo de evolução da doença, 27,08\% relataram ter descoberto sua doença há menos de três meses e $27,08 \%$ há mais de cinco anos. Os pacientes internados nas enfermarias da Clínica Médica representaram 57,29\% dos entrevistados; os da Clínica Cirúrgica, 27,08\%; e os da Unidade de Emergência, 11,46\%.

\section{Diferenças entre o atendimento do HC-UFG e outros hospitais}

A maioria dos entrevistados $(78,13 \%)$ afirmou que existe diferença entre o atendimento recebido no HC-UFG em relação ao recebido no hospital de sua última internação (fora do HC). A diferença foi quantificada como grande por $70,67 \%$ dos que notaram sua existência.

Na atribuição de notas, os outros hospitais receberam $28,26 \%$ de notas ruins, $28,26 \%$ de notas regulares, $20,65 \%$ de notas boas e 22,83\% de notas muito boas. A média global foi de 5,67 $\pm 3,38$.

O Hospital das Clínicas da UFG recebeu apenas 2,17\% de notas ruins. Notas muito boas foram atribuídas por $70,65 \%$ dos entrevistados. A boa avaliação do Hospital das Clínicas (notas boas ou muito boas) somou $93,48 \%$ das respostas. A média global foi de $9,01 \pm 1,5$ (Tabela 1 ).

A aplicação do teste de Wilcoxon comprovou que a média das notas atribuídas ao HC-UFG é estatisticamente diferente $(\mathrm{p}<0,001)$ da média das notas atribuídas ao último hospital em que o paciente esteve internado. 
TABELA 1

Notas atribuídas aos hospitais pelos pacientes

\begin{tabular}{ccccc}
\hline & \multicolumn{2}{c}{$\begin{array}{c}c \\
\text { Nospital da última } \\
\text { internação }\end{array}$} & HU \\
\cline { 2 - 5 } & $\mathrm{n}$ & 28,26 & $\mathrm{n}$ & $\%$ \\
Ruim & 26 & 28,26 & 4 & 2,17 \\
Regular & 26 & 20,65 & 21 & 4,35 \\
Boa & 19 & 22,83 & 65 & 22,83 \\
Muito boa & 21 & 100,00 & 92 & 70,65 \\
Total & 92 & 4,17 & 4 & 100,00 \\
Não soube informar & 4 & & 4,17 \\
\hline
\end{tabular}

\section{Comparação entre o atendimento no $\mathrm{HU}$ e no último} hospital em que o paciente esteve internado

O item limpeza e higiene foi referido como melhor no HCUFG por 51,04\% dos pacientes; da mesma forma, 53,13\% afirmaram que a alimentação no hospital-escola é melhor que a dos outros hospitais em que estiveram internados.

A assistência geral no HC-UFG foi avaliada como melhor por $67,61 \%$ dos entrevistados, assim como os cuidados da enfermagem $(65,63 \%)$ e o atendimento médico $(69,79 \%)$.
A estrutura física e a disponibilidade dos recursos do hospital também foram mais bem avaliadas por $55,21 \%$ e $69,79 \%$ dos pacientes, respectivamente (Tabela 2).

A aplicação do teste Regra de Sinais de Descartes mostra que a diferença entre as avaliações positivas e negativas de cada um dos itens é estatisticamente significante $(\mathrm{p}<0,001)$ Isso nos possibilita afirmar que, em todos os quesitos avaliados, o serviço do HC-UFG foi superior ao do último hospital em que o paciente esteve internado.

TABELA 2

Avaliação do HU comparado ao último hospital

\begin{tabular}{|c|c|c|c|c|c|c|c|c|}
\hline \multirow{3}{*}{ Fator avaliado } & \multicolumn{6}{|c|}{ Avaliação } & \multirow[b]{3}{*}{$\mathrm{z}$} & \multirow[b]{3}{*}{$\mathrm{p}^{*}$} \\
\hline & \multicolumn{2}{|c|}{ pior } & \multicolumn{2}{|c|}{ igual } & \multicolumn{2}{|c|}{ melhor } & & \\
\hline & $\mathrm{n}$ & $(\%)$ & $\mathrm{n}$ & $(\%)$ & $\mathrm{n}$ & $(\%)$ & & \\
\hline Limpeza/higiene $(n=96)$ & 15 & 15,63 & 32 & 33,33 & 49 & 51,04 & $-4,125$ & $<0,001$ \\
\hline Alimentação $(n=96)$ & 14 & 14,58 & 31 & 32,29 & 51 & 53,13 & $-4,465$ & $<0,001$ \\
\hline $\begin{array}{l}\text { Cuidados da enfermagem }(\mathrm{n}= \\
96)\end{array}$ & 5 & 5,21 & 28 & 29,17 & 63 & 65,63 & $-6,912$ & $<0,001$ \\
\hline Atendimento médico $(n=96)$ & 2 & 2,08 & 27 & 28,13 & 67 & 69,79 & $-7,705$ & $<0,001$ \\
\hline Assistência geral $(\mathrm{n}=96)$ & 6 & 6,25 & 25 & 26,04 & 65 & 67,71 & $-6,883$ & $<0,001$ \\
\hline Estrutura física $(\mathrm{n}=96)$ & 19 & 19,79 & 24 & 25,00 & 53 & 55,21 & $-3,889$ & $<0,001$ \\
\hline Recursos do hospital $(\mathrm{n}=96)$ & 10 & 10,42 & 19 & 19,79 & 67 & 69,79 & $-6,382$ & $<0,001$ \\
\hline
\end{tabular}

* Teste utilizado: Regra de Sinais de Descartes.

\section{Aquisição de informações sobre a doença}

Mais da metade dos pacientes (58,33\%) acha que houve aumento da informação acerca de sua doença após a internação no HC-UFG, enquanto somente 32,29\% não notaram alteração. Essa diferença foi estatisticamente significante $(p<$ $0,001)$ quando aplicada a Regra de Sinais de Descartes.

A informação adquirida foi avaliada como útil (62,5\%) e muito útil $(30,36 \%)$ pela maior parte dos entrevistados. Os motivos mais citados como responsáveis pela aquisição da informação foram: o esclarecimento por parte do médico responsável, relatado por $48,21 \%$ dos entrevistados e o esclarecimento por parte de toda a equipe hospitalar, referido por $39,29 \%$. Outro motivo também citado foi o esclarecimento por parte dos alunos $(24,21 \%)$.

\section{Avaliação da presença dos alunos no HU}

A presença dos alunos no hospital universitário foi avaliada como boa por $64,58 \%$ dos pacientes e muito boa por $27 \%$ deles 
(Tabela 3). Somente um indivíduo entrevistado avaliou como ruim a presença dos alunos no $\mathrm{HU}$. Os sentimentos despertados nos pacientes pela presença dos alunos nas enfermarias foram, em sua maioria, positivos $(84,54 \%$ das respostas), sendo eles: receptividade $(65,62 \%)$, alegria $(62,5 \%)$ e segurança (54,17\%). Os sentimentos negativos - raiva, insegurança, impaciência e incômodo - somaram $10,14 \%$ das respostas, e $11,46 \%$ disseram ser indiferentes aos alunos (Figura 1).

TABELA 3

Avaliação da presença dos alunos no HC-UFG

\begin{tabular}{lcc}
\hline \multirow{2}{*}{\begin{tabular}{l} 
Avaliação da presença \\
\cline { 2 - 3 } \multicolumn{1}{c}{ dos alunos }
\end{tabular}} & \multicolumn{2}{c}{ Número de pacientes } \\
\hline Muito boa & 26 & $(\%)$ \\
Boa & 62 & 27,08 \\
Regular & 7 & 64,58 \\
Ruim & 1 & 7,29 \\
Total & 96 & 1,04 \\
\hline
\end{tabular}

Avaliação das atividades de ensino nas enfermarias e considerações sobre invasão de privacidade

As aulas ministradas à beira do leito foram consideradas agradáveis por $69,79 \%$ dos pacientes. Somente $6,25 \%$ as acharam desagradáveis e 23,96\% foram indiferentes (Tabela 4). Comparando com o último hospital em que estiveram internados, 76,04\% dos entrevistados disseram não se considerar invadidos em sua privacidade no HU (Figura 2).

FIGURA 1

Sentimentos despertados pelos alunos nos pacientes.

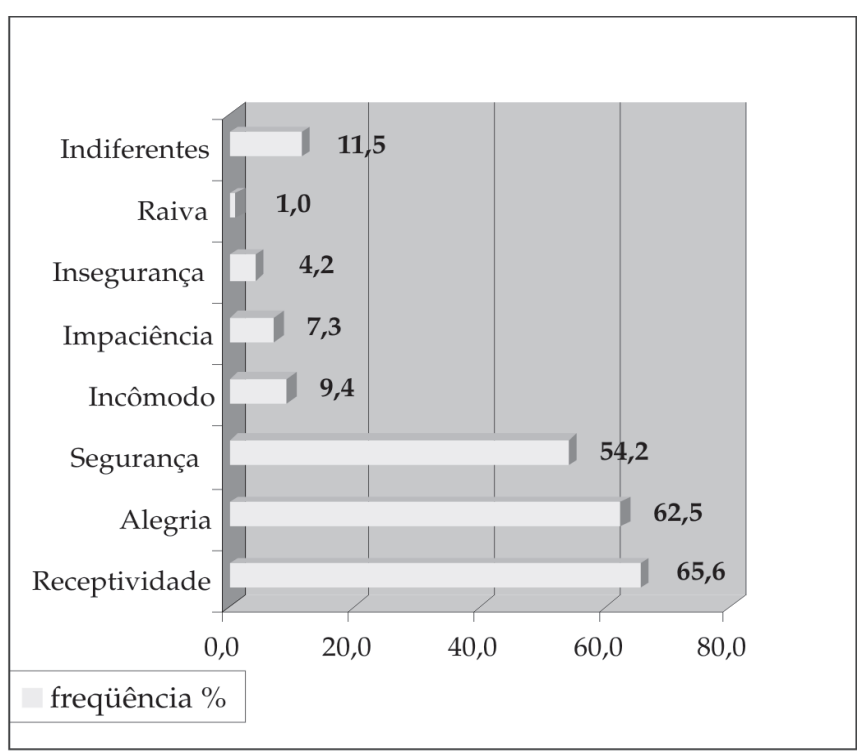

TABELA 4

Avaliação das atividades de ensino presenciadas pelos pacientes

\begin{tabular}{lcc}
\hline \multirow{2}{*}{$\begin{array}{c}\text { Avaliação das atividades de } \\
\text { ensino }\end{array}$} & $\mathrm{N}$ & Número de pacientes \\
\cline { 2 - 3 } & 67 & $(\%)$ \\
\hline Agradáveis & 6 & 69,79 \\
Desagradáveis & 23 & 6,25 \\
Indiferente & 96 & 23,96 \\
Total & & 100,00 \\
\hline
\end{tabular}


FIGURA 2.

Invasão de privacidade.

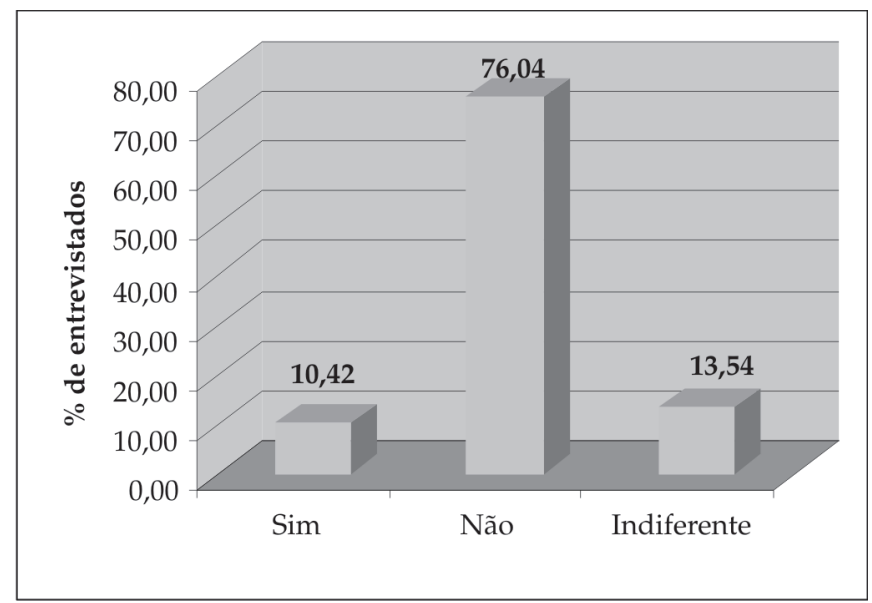

Influência do sexo e idade dos pacientes na percepção global da presença dos alunos no ambiente hospitalar

Não se encontrou diferença significativa ao se compararem as avaliações da presença dos alunos feitas pelo grupo etário entre 61 e 80 anos com as do grupo do outro extremo de idade (18 ?- 31 anos) ( $p=0,620)$. Também

\section{Número de internações no HU e aquisição de informações sobre a doença}

O aumento do número de internações no hospital-escola parece não determinar ganho de informação por parte do paciente sobre sua doença $(\mathrm{p}=0,426)$. No entanto, não houve diferença estatisticamente significante entre a percepção desses dois grupos quanto às atividades de ensino presenciadas por eles $(p=0,122)$.

O sexo dos pacientes também não foi determinante de diferenças na avaliação da presença dos alunos no hospital $(\mathrm{p}=0,439)$.

observamos que $75 \%$ dos pacientes com mais de três internações no HC-UFG relataram aquisição de informação acerca de sua doença, enquanto no grupo de entrevistados com menos de três internações esse índice representou $55,95 \%$ (Tabela 5).

TABELA 5

Número de internações no HU e aquisição de informação sobre a doença

\begin{tabular}{|c|c|c|c|c|c|c|c|}
\hline \multirow{3}{*}{$\begin{array}{c}\text { Número de } \\
\text { internações no } \\
\text { HC }\end{array}$} & \multicolumn{7}{|c|}{ Aquisição de informação } \\
\hline & \multicolumn{2}{|c|}{ Sim } & \multicolumn{2}{|c|}{ Não } & \multicolumn{2}{|c|}{ Indiferente } & \multirow{2}{*}{$\frac{\text { Total }}{\mathrm{n}}$} \\
\hline & $\mathrm{n}$ & $(\%)$ & $\mathrm{n}$ & $(\%)$ & $\mathrm{N}$ & $(\%)$ & \\
\hline$<3$ & 47 & 55,95 & 29 & 34,52 & 8 & 9,52 & 84 \\
\hline
\end{tabular}

Relação entre a nota atribuída ao HU e a avaliação da presença dos alunos e das atividades de ensino na enfermaria

Os pacientes que deram as menores notas ao serviço do hospital (ruim ou regular) avaliaram a presença dos alunos como boa e muito boa, e consideraram agradáveis ou indiferentes as atividades de ensino nas enfermarias. Dos pacientes que deram notas muito boas, $92,31 \%$ consideraram como boa ou muito boa a presença dos estudantes; $78,46 \%$ destes acharam agradáveis as atividades nas enfermarias (Tabelas 6 e 7). 
TABELA 6

Nota atribuída ao HU e avaliação da presença dos alunos

\begin{tabular}{ccccccccccc}
\hline \multirow{2}{*}{ Nota atribuída ao HU } & \multicolumn{8}{c}{ Avaliação da presença dos alunos } \\
\cline { 2 - 10 } & \multicolumn{1}{c}{ Muito boa } & \multicolumn{1}{c}{ Boa } & \multicolumn{2}{c}{ Regular } & \multicolumn{2}{c}{ Ruim } & Total \\
\cline { 2 - 10 } & $\mathrm{n}$ & $(\%)$ & $\mathrm{N}$ & $(\%)$ & $\mathrm{N}$ & $(\%)$ & $\mathrm{n}$ & $(\%)$ & \\
Ruim & 1 & 50,00 & 1 & 50,00 & - & 0,00 & - & 0,00 & 2 \\
Regular & - & 0,00 & 4 & 100,00 & - & 0,00 & - & 0,00 & 4 \\
Boa & 6 & 30,00 & 12 & 60,00 & 2 & 10,00 & - & 0,00 & 20 \\
Muito boa & 18 & 27,69 & 42 & 64,62 & 5 & 7,69 & - & 0,00 & 65 \\
Não soube informar & - & 0,00 & 3 & 75,00 & - & 0,00 & 1 & 25,00 & 4 \\
\hline
\end{tabular}

TABELA 7

Nota atribuída ao HU e avaliação das atividades de ensino na enfermaria

\begin{tabular}{cccccccc}
\hline \multirow{2}{*}{$\begin{array}{c}\text { Nota atribuída } \\
\text { ao HU }\end{array}$} & \multicolumn{7}{c}{ Atividades de ensino na enfermaria } \\
\cline { 2 - 7 } & \multicolumn{2}{c}{ Agradáveis } & \multicolumn{2}{c}{ Desagradáveis } & Indiferentes & Total \\
\cline { 2 - 7 } & $\mathrm{n}$ & $(\%)$ & $\mathrm{n}$ & $(\%)$ & $\mathrm{n}$ & $(\%)$ & $\mathrm{n}$ \\
\hline Ruim & 1 & 50,00 & - & 0,00 & 1 & 50,00 & 2 \\
Regular & 2 & 50,00 & - & 0,00 & 2 & 50,00 & 4 \\
Boa & 11 & 52,38 & 3 & 14,29 & 7 & 33,33 & 21 \\
Muito boa & 51 & 78,46 & 3 & 4,62 & 11 & 16,92 & 65 \\
Não soube informar & 2 & 50,00 & - & 0,00 & 2 & 50,00 & 4
\end{tabular}

\section{DISCUSSÃO}

Nosso estudo mostrou que, de maneira geral, os pacientes avaliaram o atendimento no HU como muito bom. A qualidade desse atendimento envolve uma parte técnica, inerente ao funcionamento de um hospital-escola, e outra parte representada pelas relações humanas, também de fundamental importância na recuperação dos pacientes.

Na grande maioria dos hospitais, a equipe multidisciplinar de profissionais de saúde trabalha quase sempre de maneira independente, no intuito de proporcionar melhores cuidados aos pacientes. Em um hospital-escola, essa equipe tem uma atuação mais coesa e ainda conta com a participação dos estudantes, que, segundo os resultados deste estudo, é bastante significativa.

Foi demonstrado que os pacientes do HU estão satisfeitos com a presença dos alunos no hospital e que estes lhes despertam sentimentos de alegria, segurança e acolhimento. Isto reforça a importância do estudante no meio hospitalar, ao evidenciar sua influência positiva no atendimento.

Uma limitação do estudo deveu-se ao fato de os questionários terem sido aplicados por alunas da Faculdade de Medicina que freqüentam o HU. Isso poderia ter induzido os pacientes a avaliar positivamente a presença dos alunos, as aulas na enfermaria e os serviços oferecidos pelo HC-UFG. O grupo pesquisador tentou minimizar esse viés, conscientizando os entrevistados de que os questionários eram anônimos e que de maneira alguma haveria repercussões no tratamento por eles recebido. Além disso, os resultados foram de tal maneira significativos, que praticamente impossibilitaram um viés dessa magnitude.

Outra limitação foi a realização do estudo somente nas enfermarias de longa permanência do HC-UFG, o que pode representar apenas a realidade de um setor de atendimento. Este viés, entretanto, não invalida nossos achados específicos.

Estudo conduzido por Simon et al. ${ }^{5}$, desenvolvido em um centro de saúde que recebe alunos da Faculdade de Medicina de Harvard, mostrou que as instruções dadas por médicos a alunos durante os atendimentos ambulatoriais não afetam a satisfação dos pacientes. Em nossos dados, pelo contrário, a maioria dos entrevistados considerou agradáveis as atividades de ensino nas enfermarias. Apesar de estes serem aspectos que não representam obrigatoriamente o mesmo sentimento, há uma aproximação entre estas avaliações, e, por este motivo, estes dados podem ser comparados.

Essa diferença na avaliação pode ser devida às características socioeconômicas e culturais da população assistida nos hospitais públicos brasileiros ou à melhor qualidade da relação com o paciente por nós estabelecida. Também não se deve esquecer que a diferença pode estar relacionada ao local da pesquisa. 
Hajioff e Birchall4 observaram que a presença dos estudantes nos atendimentos ambulatoriais de dois hospitaisescola não interfere na satisfação dos pacientes com o serviço. Em contrapartida, nosso estudo mostrou que os pacientes internados no Hospital das Clínicas da Universidade Federal de Goiás estão bastante satisfeitos com a presença dos alunos como participantes de seu atendimento. Até que ponto este fato, a exemplo do observado no parágrafo acima, se deve ao local da pesquisa (enfermaria) é algo que não podemos analisar.

Como esperado, o ambiente acadêmico traz ao paciente mais informações a respeito de sua doença. Foi agradável perceber que a maioria dos entrevistados considerou boa a qualidade da informação adquirida nas enfermarias do hospital, seja por informações prestadas pela equipe, seja pela vivência das atividades acadêmicas.

Quanto aos aspectos técnicos, relacionados às características hospitalares propriamente ditas, o estudo mostrou que a nota média recebida pelo $H U$ foi consideravelmente maior do que a média atribuída ao hospital da última internação do paciente. Isso significa que, apesar das dificuldades e do sucateamento enfrentados por um hospital público, o HC-UFG foi bem avaliado pela maioria dos pacientes em todos os quesitos investigados.

É interessante notar que mesmo os pacientes que deram as menores notas ao serviço do hospital (ruim e regular) avaliaram a presença dos alunos como boa e muito boa, e consideraram agradáveis as atividades nas enfermarias. Dessa forma, o ensino médico no hospital não foi o fator responsável pela insatisfação com o serviço.

A pesquisa demonstrou que o aluno tem papel de grande relevância no atendimento prestado no hospital-escola. Ele contribui para o aumento da confiança no serviço, desperta maior segurança e alegria nos pacientes, torna o ambiente mais acolhedor e atua como disseminador de informações.
Todos esses fatores são fundamentais para a formação dos novos médicos da Universidade Federal de Goiás, já que oferecem aos acadêmicos a possibilidade de compreender que a atuação médica transcende os aspectos exclusivamente técnicos. A conseqüência direta deste fato é a melhoria da qualidade do atendimento prestado à população.

\section{REFERÊNCIAS}

1. Martins C. O estudante de medicina frente ao paciente. In: Perspectivas da relação médico-paciente. 2. ed. Porto Alegre: Artes Médicas; 1981. p.183-187.

2. Porto CC. Exame clínico: bases para prática médica. 5. ed. Rio de Janeiro: Guanabara Koogan; 2004.

3. Gresst W. Effect of students envolvement on patient perceptions of ambulatory care visits: a randomized controlled trial. J Gen Intern Med 2002;17(6):420-7.

4. Hajioff D, Birchall M. Medical students in ENT outpatient clinics: appointment times, patient satisfaction and student satisfaction. Med Educ 1999; 33:669-73.

5. Simon SR, Peters AS, Christiansen CL, Fletcher RH. Effect of medical student teaching on patient satisfaction in a manage care setting. J Gen Intern Med 2000; 15:457-61.

\section{CONFLITOS DE INTERESSE}

Declarou não haver

\section{ENDEREÇO PARA CORRESPONDÊNCIA}

Universidade Federal de Goiás

Faculdade de Medicina - Liga de Hipertensão Arterial $1^{\text {a }}$ Avenidas/n St. Universitáriao - Campus I UFG 74605-020 - Goiânia - Goiás

Fone: (62) 3209-6149

e-mail fvjardim@medicina.ufg.br 